\title{
ZERO DISTRIBUTION AND FACTORIZATION OF ANALYTIC FUNCTIONS OF SLOW GROWTH IN THE UNIT DISC
}

\author{
I. CHYZHYKOV
}

(Communicated by Mario Bonk)

\begin{abstract}
For a meromorphic function $f$ in the unit disc, let the $\rho_{\infty}$-order of the growth be the limit of the orders of $L_{p}$-norms of $\log \left|f\left(r e^{i \theta}\right)\right|$ over the circle. In the case when the order of the maximum modulus function is smaller than 1 , we describe zero distribution of canonical products and derive a new factorization theorem and logarithmic derivative estimates.
\end{abstract}

\section{INTRODUCTION AND THE MAIN RESULT}

Let $D(z, t)=\{\zeta \in \mathbb{C}:|\zeta-z|<t\}, z \in \mathbb{C}, t>0$, and $\mathbb{D}=D(0,1)$. For an analytic function $f$ in $\mathbb{D}$, we define the maximum modulus $M(r, f)=\max \{|f(z)|$ : $|z|=r\}, 0 \leq r<1$. In the sequel, the symbol $C$ with indices stands for positive constants which depend on the parameters indicated. We write $a(r) \sim b(r)$ if $\lim _{r \uparrow 1} a(r) / b(r)=1$.

Usually, the orders of growth of an analytic function $f$ in $\mathbb{D}$ are defined as

$$
\rho_{M}[f]=\limsup _{r \uparrow 1} \frac{\log ^{+} \log ^{+} M(r, f)}{-\log (1-r)}, \rho_{T}[f]=\limsup _{r \uparrow 1} \frac{\log ^{+} T(r, f)}{-\log (1-r)},
$$

where $T(r, f)=\frac{1}{2 \pi} \int_{0}^{2 \pi} \log ^{+}\left|f\left(r e^{i \theta}\right)\right| d \theta$. It is well known that

$$
\rho_{T}[f] \leq \rho_{M}[f] \leq \rho_{T}[f]+1,
$$

and all admissible values of the orders are possible ([1, 2], 10]).

Many theorems on analytic functions in $\mathbb{D}$ fail to hold when the $\rho_{M}$ order is smaller than 1 (see e.g. [2], 9], 11]). To be more precise we start with canonical products. Let $A=\left(a_{n}\right)$ be a sequence of complex numbers in $\mathbb{D}$ without accumulation points in $\mathbb{D}$. We define the exponent of convergence of $A$ by $(\inf \emptyset=+\infty)$

$$
\mu[A]=\inf \left\{\mu \geq 0: \sum_{a_{n} \in A}\left(1-\left|a_{n}\right|\right)^{\mu+1}<\infty\right\} .
$$

Received by the editors May 9, 2011 and, in revised form, August 9, 2011.

2010 Mathematics Subject Classification. Primary 30J99; Secondary 30D35, 30H15, 37A45.

Key words and phrases. Analytic function, factorization, zero distribution, canonical product, proximate order, order of growth, logarithmic derivative. 
It is well known [4, 13, 15] that the Džrbašjan-Naftalevich-Tsuji canonical product

$$
P(z, A, q)=\prod_{n=1}^{\infty} E\left(\frac{1-\left|a_{n}\right|^{2}}{1-\bar{a}_{n} z}, q\right),
$$

where $E(w, 0)=(1-w)$,

$$
E(w, q)=(1-w) \exp \left\{w+w^{2} / 2+\cdots+w^{q} / q\right\}, \quad q \in \mathbb{N},
$$

is an analytic function with the zero sequence $A$ provided that $\sum_{a_{n} \in A}\left(1-\left|a_{n}\right|\right)^{q+1}<$ $\infty$.

C. N. Linden [9] established a connection between $\rho_{M}[P]$ and the zero distribution of $P$, where $P$ is of the form (1.2). To clarify this connection we need some definitions.

Let

$$
\square\left(r e^{i \varphi}\right)=\left\{\zeta: r \leq|\zeta| \leq \frac{1+r}{2},|\arg \zeta-\varphi| \leq \frac{1-r}{4}\right\},
$$

and $\nu\left(r e^{i \varphi}\right)$ be the number of zeros of $P$ in $\square\left(r e^{i \varphi}\right)$. We define

$$
\nu_{1}(r, P)=\max _{\varphi} \nu\left(r e^{i \varphi}\right), \quad \nu[P]=\limsup _{r \uparrow 1} \frac{\log ^{+} \nu_{1}(r, P)}{-\log (1-r)},
$$

and

$$
\rho_{n}[P]=\limsup _{r \uparrow 1} \frac{\log ^{+} n(r, P)}{-\log (1-r)},
$$

where $n(r, P)$ is the number of zeros in $\bar{D}(0, r)$.

Theorem A ([9, Theorem V]). With the notation above we have

$$
\begin{aligned}
& \rho_{T}[P]=\left(\rho_{n}[P]-1\right)^{+}, \\
& \rho_{M}[P] \begin{cases}=\nu[P], & \rho_{M}[P] \geq 1, \\
\leq \nu[P] \leq 1, & \rho_{M}[P]<1 .\end{cases}
\end{aligned}
$$

Remark 1.1. We note that relation (1.6) follows essentially from [13, 15]. Moreover, $\left(\rho_{n}[P]-1\right)^{+}$is equal to the convergence exponent of the zero sequence of $P$.

A function $\rho:[0,1) \rightarrow \mathbb{R}_{+}$is called a proximate order $([6$, p. 55]; cf. [8] $)$ if it satisfies the following conditions:

(i) $\rho$ is differentiable on $[0,1)$;

(ii) $\lim _{r \uparrow 1} \rho(r)=\rho_{0} \in[0, \infty)$;

(iii) $\lim _{r \uparrow 1} \rho^{\prime}(r)(1-r) \log (1-r)=0$.

An advantage of this definition is that for every analytic function $f$ of finite positive order $\rho_{M}[f]$ there exists a proximate order $\rho(r)$ such that

$$
\limsup _{r \uparrow 1}(1-r)^{\rho(r)} \log M(r, f)=1 .
$$

Linden's proof of Theorem $\mathrm{A}$ is based on the following lemma [9, Lemma I], which we formulate in a suitable form for our purposes.

Lemma B. Let $\rho:[0,1) \rightarrow \mathbb{R}_{+}$be a proximate order, $\rho(r) \rightarrow \rho>0(r \uparrow 1)$. Let $P_{s}(z)=P(z, A, s)$ be a canonical product. Suppose that for some $C_{1}>0$, we have

$$
\nu\left(r e^{i \varphi}\right) \leq \frac{C_{1}}{(1-r)^{\rho(r)}}, \quad 0 \leq r<1,0 \leq \varphi<2 \pi,
$$


and $s>\rho$. Then

$$
\log \left|P_{s}(z)\right| \leq 2^{s+2} \sum_{n=1}^{\infty}\left|\frac{1-\left|a_{n}\right|^{2}}{1-z \bar{a}_{n}}\right|^{s+1} \leq \frac{C_{2}}{(1-|z|)^{\rho(r)}}, \quad z \in \mathbb{D},
$$

for some constant $C_{2}>0$.

Remark 1.2. Linden proved the lemma for the case $\rho(r) \equiv \rho$, but the same proof works in the general case as well (cf. [6, Chaps. 2, 3]).

As we can see from Theorem $\mathrm{A}$, the value $\nu[P]$ coincides with $\rho_{M}[P]$ when the order is greater than 1 . The question arises:

Question 1.3. What kind of growth characteristic can describe zero distribution in the case when $\rho_{M}[f] \leq 1$ ?

For a meromorphic function $f(z), z \in \mathbb{D}$, and $p \geq 1$ we define

$$
m_{p}(r, f)=\left(\left.\frac{1}{2 \pi} \int_{0}^{2 \pi}|\log | f\left(r e^{i \theta}\right)\right|^{p} d \theta\right)^{\frac{1}{p}}, \quad 0<r<1 .
$$

We write

$$
\rho_{p}[f]=\limsup _{r \uparrow 1} \frac{\log m_{p}(r, f)}{-\log (1-r)} .
$$

A characterization of $\rho_{p}$-orders can be found in [12].

We define the $\rho_{\infty}$-order of $f$ as

$$
\rho_{\infty}[f]=\lim _{p \rightarrow \infty} \rho_{p}[f]
$$

(existence of the limit follows from the fact that $L_{p}$-norms are monotone in $p$ ). It follows from the First Fundamental Theorem of Nevanlinna that $\rho_{1}[f]=\rho_{T}[f]$. Besides, it is known (e.g. [11]) that $\rho_{M}[f] \leq \rho_{p}[f]+\frac{1}{p}(p>0)$, which generalizes (1.1). Consequently, $\rho_{M}[f] \leq \rho_{\infty}[f]$. Moreover, Linden [11] proved that $\rho_{\infty}[f]=\rho_{M}[f]$ provided that $\rho_{M}[f] \geq 1$. Thus, the values $\rho_{\infty}[f]$ and $\nu[f]$ have similar behavior with respect to the maximum modulus order when $f$ is a canonical product. The main purpose of this paper is to prove that $\rho_{\infty}[f]$ coincides with $\nu[f]$ for the canonical products.

For a sequence $A$ in $\mathbb{D}$ with the finite convergence exponent we define $\nu[A]=$ $\nu[P(z, A, q)]$ for an appropriate choice of $q$. It is clear that the definition does not depend on $q$.

Theorem 1.4. Given a sequence $A=\left(a_{k}\right)$ in $\mathbb{D}$ such that $\nu=\nu[A]<\infty$ and an integer $s \geq[\nu]+1$, we define the canonical product $P_{s}(z)=P(z, A, s)$. Then $\rho_{\infty}\left[P_{s}\right]=\nu$.

Theorem 1.5. Let $f$ be an analytic function in $\mathbb{D}$. Then $\nu[f] \leq \rho_{\infty}[f]$.

Example 1.6. Let $a_{k}=1-1 /\left(k \log ^{2} k\right), k \in\{3, \ldots\}$. We consider the canonical product $B(z)=P(z, A, 0)$, which is a Blaschke product up to a constant factor. Since $|B|$ is bounded in $\mathbb{D}$, we have $\rho_{M}[B]=\rho_{T}[B]=0$, and consequently $\rho_{\infty}[B] \leq 1$.

On the other hand, it is easy to check that

$$
n(r, B) \sim \frac{1}{(1-r) \log ^{2}(1-r)}, \quad r \uparrow 1,
$$


and

$$
\frac{d_{1}}{(1-r) \log ^{2}(1-r)} \leq \nu(r) \leq \frac{d_{2}}{(1-r) \log ^{2}(1-r)}, \quad r \uparrow 1,
$$

for some positive constants $d_{1}, d_{2}$. Hence, $\nu[B]=1$, and by Theorem $1.5, \rho_{\infty}[B]=1$.

In Section 2 we prove Theorem 1.4, which is the main result of the paper. In Section 3 we apply the theorem to problems of factorization of analytic and meromorphic functions in $\mathbb{D}$ and derive some new logarithmic derivative estimates.

\section{Proofs of Theorems 1.4 And 1.5}

We write $\rho=\rho_{\infty}\left[P_{s}\right]$.

First, we show that $\nu \leq \rho$. The proof of this inequality is based on ideas from [8, Chap. 2, Lemmas 10, 11].

Lemma 2.1. Under the assumptions of Theorem 1.4 we have

$$
\limsup _{r \uparrow 1} \frac{\sup _{\varphi} \log \log \inf _{\zeta \in \square\left(r e^{i \varphi}\right)} \frac{1}{\left|P_{s}(\zeta)\right|}}{\log \frac{1}{1-r}} \leq \rho .
$$

Note that inequality (2.1) is a counterpart of Levin's condition (see [8, Chap. 2]), which plays an important role in the theory of subharmonic functions in the halfplane ([5, Theorem 18]) and the theory of functions of completely regular growth in an angle [8].

Proof of Lemma 2.1. Suppose that (2.1) does not hold; i.e., there exist $\varepsilon>0$, a sequence $\left(r_{n}\right)$ tending to 1 , and a sequence $\left(\varphi_{n}\right)$ such that

$$
\log \frac{1}{\left|P_{s}(\zeta)\right|} \geq \frac{1}{(1-|\zeta|)^{\rho+\varepsilon}}
$$

for all $\zeta \in \square\left(r_{n} e^{i \varphi_{n}}\right)$. Then

$$
\left.\int_{\varphi_{n}-\frac{1-r_{n}}{4}}^{\varphi_{n}+\frac{1-r_{n}}{4}}|\log | P_{s}\left(r_{n} e^{i \theta}\right)\right|^{p} d \theta \geq \frac{1-r_{n}}{2\left(1-r_{n}\right)^{(\rho+\varepsilon) p}} .
$$

Hence, $m_{p}\left(r_{n}, P_{s}\right) \geq\left(\frac{1}{4 \pi}\right)^{\frac{1}{p}}\left(1-r_{n}\right)^{-\rho-\varepsilon+\frac{1}{p}}$. Thus, $\rho_{p}\left[P_{s}\right] \geq \rho+\varepsilon-\frac{1}{p}$. Passing to the limit as $p \rightarrow \infty$, we obtain $\rho_{\infty}\left[P_{s}\right] \geq \rho+\varepsilon$. This contradiction proves (2.1).

If $\rho>0$, then the inequality $\nu \leq \rho$ follows from $\rho_{M}\left[P_{s}\right] \leq \rho$ and the next lemma.

Lemma 2.2. Let $f$ be an analytic function in $\mathbb{D}, \rho:[0,1) \rightarrow \mathbb{R}_{+}$be a proximate order, $\rho(r) \rightarrow \rho>0(r \uparrow 1)$. Let

$$
\log \left|f\left(r e^{i \varphi}\right)\right| \leq C_{3}(1-r)^{-\rho(r)}, \quad r \in[0,1)
$$

for some $C_{3}>0$.

If there exist $N>0$ and $r_{0} \in(0,1)$ such that for all $r \in\left[r_{0}, 1\right)$ and $\varphi \in[0,2 \pi)$ and some $z^{*} \in \square\left(r e^{i \varphi}\right)$ we have

$$
\log \left|f\left(z^{*}\right)\right|>-\frac{N}{(1-r)^{\rho(r)}}
$$

then

$$
\nu\left(r e^{i \varphi}\right) \leq C_{4} \frac{C_{3}+N}{(1-r)^{\rho(r)}}, \quad r \uparrow 1,
$$

where $C_{4}$ is an absolute constant. 
Proof of Lemma 2.2. Without loss of generality we may assume that $\varphi=0$. Let $r \in[0,1)$. We write $R=r+(1-r) / 4, \varphi_{r}=(1-r) / 4$. Then $1-R=\frac{3}{4}(1-r)$. Since

$$
\varphi_{r}^{2}+\left(\frac{1+r}{2}-R\right)^{2}=\frac{1}{8}(1-r)^{2},
$$

and $\frac{1}{2 \sqrt{2}}<\frac{2}{5}$, we have

$$
\square(r) \subset D\left(R, \frac{2}{5}(1-r)\right), \quad r \in\left[r_{1}, 1\right)
$$

for some $r_{1} \in[0,1)$. that

$$
\log \left|f\left(z_{r}\right)\right|>-\frac{N}{\left(1-\left|z_{r}\right|\right)^{\rho\left(\left|z_{r}\right|\right)}} \geq-\frac{N}{\left(\frac{1-r}{4}\right)^{\rho\left(1-\frac{1-r}{4}\right)}},
$$

because $\left|z_{r}\right| \leq 1-\frac{1-r}{4}$. On the other hand, we have

$$
\log |f(z)| \leq \frac{C_{3}}{\left(\frac{1-r}{4}\right)^{\rho\left(1-\frac{1-r}{4}\right)}}, \quad z \in D\left(R, \frac{1-r}{2}\right) .
$$

Therefore the function $\psi_{r}(z):=\frac{f(z+R)}{f\left(z_{r}\right)}$ is analytic in $D\left(0, \frac{1-r}{2}\right)$. We also have $\psi_{r}\left(z_{r}-R\right)=1$, and

$$
\log \left|\psi_{r}(z)\right| \leq\left(C_{3}+N\right)\left(\frac{1-r}{4}\right)^{-\rho\left(1-\frac{1-r}{4}\right)}, \quad|z| \leq \frac{1-r}{2}
$$

by (2.3) and (2.4).

We need the following lemma [8, Chap. 2, Lemma 9].

Lemma C. Let $\Phi$ be an analytic function in $D\left(0, R_{0}\right)$, and let $z_{0} \in \bar{D}\left(0, l R_{0}\right)$, where $l<1$, be such that $\left|\Phi\left(z_{0}\right)\right|=1$. Then

$$
n\left(t R_{0}, \Phi\right) \leq C_{5}(l, t) \log M\left(R_{0}, \Phi\right), \quad l<t<1,
$$

where $C_{5}(l, t)=\left(\log \frac{1+l t}{l+t}\right)^{-1}$.

Applying Lemma C] with $R_{0}=(1-R) / 2, l=\frac{2}{5}: \frac{1}{2}=0.8$, we get

$$
\nu(r) \leq n\left(D\left(R, \frac{1-r}{2}\right)\right) \leq C_{5}(0,8 ; t)\left(C_{3}+N\right)\left(\frac{1-r}{4}\right)^{-\rho\left(1-\frac{1-r}{4}\right)}, \quad 0.8<t<1 .
$$

Fixing $t \in(0.8 ; 1)$ and using the property $\left(\frac{1-r}{4}\right)^{-\rho\left(1-\frac{1-r}{4}\right)} \sim 4^{-\rho}(1-r)^{-\rho(r)}(r \uparrow 1)$, we obtain the required assertion. Lemma 2.2 is proved.

If $\rho=0$, then we can apply previous arguments with arbitrary positive $\varepsilon_{0}$ instead of $\rho(r)$ to obtain $\nu \leq \varepsilon_{0}$. Thus $\nu=0$. The inequality $\rho \leq \nu$ follows from the next lemma. Note that our proof of Lemma 2.3 essentially repeats the arguments from [11, Lemma 1].

Lemma 2.3. Let $A=\left(a_{n}\right)$ be a sequence of complex numbers in $\mathbb{D}, \rho$ be a proximate order on $[0,1), \rho(r) \rightarrow \rho_{0}(r \uparrow 1)$. Suppose that

$$
\nu\left(r e^{i \varphi}\right) \leq \frac{C_{6}}{(1-r)^{\rho(r)}}, \quad r \in[0,1), \varphi \in[0,2 \pi)
$$


for some $C_{6}>0$. If $s>\rho_{0}$, then there exists a constant $C_{7}$, independent of $r$ and $p$, such that

$$
m_{p}\left(r, P_{s}\right) \leq C_{7} \frac{\log \frac{1}{1-r}}{(1-r)^{\rho(r)}}, \quad p \geq 1, r \in[0,1) .
$$

Proof of Lemma 2.3. We have to prove that

$$
\left.\int_{0}^{2 \pi}|\log | P_{s}\left(r e^{i \theta}, A\right)\right|^{p} d \theta \leq C_{7}^{p} \frac{\log ^{p} \frac{1}{1-r}}{(1-r)^{p \rho(r)}} .
$$

We deal with the integral in (2.6) by covering the range of integration by $[\pi /(1-r)]+$ 1 intervals of the form $[\tau+r-1, \tau+1-r]$ for $\tau=2 k(1-r)$ and $k \in\{0, \ldots,[\pi /(1-r)]\}$, showing that

$$
\left.\int_{\tau+r-1}^{\tau+1-r}|\log | P_{s}\left(r e^{i \theta}, A\right)\right|^{p} d \theta \leq C_{8}^{p}(1-r)^{-p \rho(r)+1} \log ^{p} \frac{1}{1-r}
$$

for each $\tau$, where the constant $C_{8}$ is independent of $\tau$. For convenience and without loss of generality, we may suppose that $\tau=0$.

We shall need some known results.

Theorem D (Tsuji, [14, Theorem V.25, p. 224]). For the canonical product $P_{s}(z)$ and positive $\varepsilon$, if $D_{m}$ denotes the disc $D\left(a_{m},\left(1-\left|a_{m}\right|^{2}\right)^{s+4}\right)$, then

$$
\log \left|P_{s}(z)\right| \geq K \log (1-|z|) \sum_{m}\left|\frac{1-\left|a_{m}\right|^{2}}{1-z \bar{a}_{m}}\right|^{s+1+\varepsilon}, \quad \frac{1}{2} \leq|z|<1, z \notin \bigcup_{m} D_{m}
$$

Without loss of generality, we assume that $\frac{3}{4} \leq\left|a_{m}\right|<1$. For given $r$, let $\gamma_{r}=\left\{z=r e^{i \theta}: r-1 \leq \theta \leq 1-r\right\}$, and $F(r)=\left\{m: D_{m} \cap \gamma_{r} \neq \emptyset\right\}$, where $D_{m}$ are the exceptional discs of Theorem D, By (2.5), we have

$$
|F(r)| \leq C_{9}(1-r)^{-\rho(r)},
$$

where $|F(r)|$ denotes the number of elements in the set $F(r)$. We consider the factorization $P_{s}=B_{1} B_{2} B_{3}$, where

$$
\begin{aligned}
& B_{1}(z)=\prod_{m \notin F(r)} E\left(\frac{1-\left|a_{m}\right|^{2}}{1-\bar{a}_{m} z}, s\right), \\
& B_{2}(z)=\prod_{m \in F(r)} \exp \sum_{j=1}^{s} \frac{1}{j}\left(\frac{1-\left|a_{m}\right|^{2}}{1-z \bar{a}_{m}}\right)^{j}, \\
& B_{3}(z)=\prod_{m \in F(r)}\left(1-\frac{1-\left|a_{m}\right|^{2}}{1-z \bar{a}_{m}}\right)=\prod_{m \in F(r)}\left(\frac{\bar{a}_{m}\left(a_{m}-z\right)}{1-z \bar{a}_{m}}\right) .
\end{aligned}
$$

First we note that for any positive number $\varepsilon$, Theorem $\mathrm{D}$ and Lemma $\mathrm{B}$ give

$$
\begin{aligned}
\int_{r-1}^{1-r}|\log | B_{1}\left(r e^{i \theta}\right)||^{p} d \theta & \leq \int_{r-1}^{1-r} C_{10}^{p} \log ^{p} \frac{1}{1-r}\left(\sum_{m}\left|\frac{1-\left|a_{m}\right|^{2}}{1-r e^{i \theta} \bar{a}_{m}}\right|^{s+1}\right)^{p} d \theta \\
& \leq C_{10}^{p} \log ^{p} \frac{1}{1-r} \frac{1}{(1-r)^{p \rho(r)}} 2(1-r)=\frac{C_{11}(\rho) \log ^{p} \frac{1}{1-r}}{(1-r)^{p \rho(r)-1}} .
\end{aligned}
$$


Next, the inequality $\left|1-z \bar{a}_{m}\right|>\frac{1}{2}\left(1-\left|a_{m}\right|^{2}\right)$ yields

$$
|\log | B_{2}(z)||<\sum_{m \in F(r)} \sum_{j=1}^{s} \frac{1}{j}\left|\frac{1-\left|a_{m}\right|^{2}}{1-z \bar{a}_{m}}\right|^{j} \leq C_{12}|F(r)| .
$$

Hence (2.9) implies that

$$
\left.\int_{r-1}^{1-r}|\log | B_{2}\left(r e^{i \theta}\right)\right|^{p} d \theta \leq C_{13}(1-r)^{1-p \rho(r)} .
$$

Finally, in [11, p. 124] it is proved that

$$
\int_{r-1}^{1-r}|\log | B_{3}\left(r e^{i \theta}\right)||^{p} d \theta \leq C_{14}|F(r)|^{p}(1-r) .
$$

Inequality (2.7) now follows from (2.10)-(2.12), so Lemma 2.3 is proved.

Proof of Theorem 1.5. We note that the proof of Lemma 2.1 is valid for any analytic function $f$ in $\mathbb{D}$. Let $A=\left(a_{k}\right)$ be the zero sequence of $f$, and $\rho>0$. Since $\rho_{M}[f] \leq \rho_{\infty}[f]$, using Lemmas 2.1 and 2.2 we deduce that $\nu[A] \leq \rho_{\infty}[f]$, i.e. $\nu[f] \leq \rho_{\infty}[f]$.

\section{Applications}

3.1. Factorization. In [9, Theorem I] Linden proved the following result.

Theorem E. Let $f$ be analytic in $\mathbb{D}$ and of order $\rho_{M}[f] \geq 1$. Then

$$
f(z)=z^{p} P(z) g(z),
$$

where $P$ is a canonical product displaying the zeros of $f, p$ is a nonnegative integer, $g$ is nonzero and both $P$ and $g$ are analytic and of $\rho_{M}$-order at most $\rho_{M}[f]$.

Further, in Theorem IV [9], Linden showed that actually

$$
\max \left\{\rho_{M}[P], \rho_{M}[g]\right\} \leq \max \left\{\rho_{M}[f], \nu[f]\right\} .
$$

Taking into account Theorem 1.5 we deduce that $\max \left\{\rho_{M}[P], \rho_{M}[g]\right\} \leq \rho_{\infty}[f]$ in this case.

Theorem 3.1. Let $f$ be analytic in $\mathbb{D}$, and of finite order $\rho_{\infty}[f]$. Then

$$
f(z)=z^{p} P(z) g(z),
$$

where $P$ is a canonical product displaying the zeros of $f, p$ is a nonnegative integer, $g$ is nonzero and both $P$ and $g$ are analytic and of $\rho_{\infty}$-order at most $\rho_{\infty}[f]$.

Proof of Theorem 3.1. Let $s=[\nu[f]]+1$. Consider the canonical product $P(z)=$ $P(z, A, s)$. This leads to the factorization $f(z)=z^{p} P_{s}(z) g(z)$, where $p$ is the multiplicity of the zero at the origin and $g$ is analytic and nonzero in $\mathbb{D}$. By Theorems 1.4 and 1.5, we have

$$
\rho_{\infty}\left[P_{s}\right]=\nu\left[P_{s}\right]=\nu[f] \leq \rho_{\infty}[f] .
$$

Since the multiplication by the factor $z^{p}$ does not change the order $\rho_{p}$ and

$$
\rho_{p}[g] \leq \max \left\{\rho_{p}[f], \rho_{p}\left[P_{s}\right]\right\} \leq \max \left\{\rho_{\infty}[f], \rho_{\infty}\left[P_{s}\right]\right\},
$$

passing to the limit in the latter inequality we get

$$
\rho_{\infty}[g] \leq \max \left\{\rho_{\infty}[f], \rho_{\infty}\left[P_{s}\right]\right\}=\rho_{\infty}[f] .
$$


The $\rho_{\infty}$-order of nonconstant meromorphic functions has the following properties:

i) $\rho_{\infty}[1 / f]=\rho_{\infty}[f]$;

ii) $\rho_{\infty}[f g] \leq \max \left\{\rho_{\infty}[f], \rho_{\infty}[g]\right\}$.

These properties yield $\rho_{\infty}[f / g] \leq \max \left\{\rho_{\infty}[f], \rho_{\infty}[g]\right\}$.

In view of the last inequality and Theorem 3.1 the next question arises naturally:

Question 3.2. Given a meromorphic function $f$ in $\mathbb{D}$ of finite order, is it possible to represent it in the form

$$
f(z)=z^{p} \frac{P(z)}{Q(z)} g(z)
$$

where $p \in \mathbb{Z}, P, Q$ are canonical products displaying zeros and poles of $f$ respectively, $g$ is a nonzero analytic function, and all $P, Q, g$ are of $\rho_{\infty}$-order at most $\rho_{\infty}[f]$ ?

It turns out that the answer is in the negative.

Theorem 3.3. There exists a meromorphic function $f$ in $\mathbb{D}$ such that $\rho_{\infty}[f]=0$, and $\nu[A]=\nu\left[A^{*}\right]=1$, where $A$ and $A^{*}$ are sequences of zeros and poles of $f$, respectively. Therefore, for any factorization of $f$ of the form (3.1), $\rho_{\infty}[P] \geq 1$, $\rho_{\infty}[Q] \geq 1$.

Proof of Theorem 3.3. Let $r_{k}=1-2^{-k}, a_{k}=r_{k}, a_{k}^{*}=a_{k}+2^{-k^{2}}, p_{k}=\left[2^{k} k^{-2}\right]$, $k \in \mathbb{N}$. Since $\sum_{k=1}^{\infty} p_{k}\left(1-a_{k}\right)<+\infty$, we can consider the Blaschke products

$$
B(z)=\prod_{k=1}^{\infty}\left(\frac{a_{k}-z}{1-z \bar{a}_{k}}\right)^{p_{k}} \equiv \prod_{k=1}^{\infty}\left(F\left(z, a_{k}\right)\right)^{p_{k}}, \quad B^{*}(z)=\prod_{k=1}^{\infty}\left(F\left(z, a_{k}^{*}\right)\right)^{p_{k}}
$$

which are analytic in $\mathbb{D}$.

Define $f(z)=B(z) / B^{*}(z)$. We shall show that $\rho_{\infty}[f]=0$, though $\rho_{\infty}[B]=$ $\rho_{\infty}\left[B^{*}\right]=1$. The latter equalities follow from Corollary 1.5 and the equalities $\nu[B]=\nu\left[B^{*}\right]=1$, which are easy to check. We are going to prove that $m_{p}(r, f)$ is bounded on $[0,1)$ for all $p>1$. This will imply that $\rho_{\infty}[f]=0$.

Let $z=r e^{i \varphi}, r \in\left[r_{m}, r_{m+1}\right)$ for some $m \in \mathbb{N}$. In order to estimate $|\log | f\left(r e^{i \varphi}\right)||$ we consider three cases.

If $\operatorname{Im} z \neq 0$, we can write

$$
|\log | F\left(z, a_{k}\right)|-\log | F\left(z, a_{k}^{*}\right)||=\left|\operatorname{Re} \int_{\left[a_{k}, a_{k}^{*}\right]} \frac{1-\left|z^{2}\right|}{(\zeta-z)(1-\bar{z} \zeta)} d \zeta\right| .
$$

If $k \leq m-2$ or $k \geq m+2$, and $\zeta$ is such that $r_{k} \leq|\zeta| \leq r_{k+1}$, then

$$
|\zeta-z| \geq r_{m+2}-r_{m+1} \geq(1-r) / 4
$$

and

$$
\frac{1-|z|^{2}}{|\zeta-z||1-z \zeta|} \leq \frac{1-|z|^{2}}{\frac{1-|z|}{4}(1-|\zeta|)}=\frac{8}{1-|\zeta|}
$$


Combining (3.3) and the latter inequality, we obtain

$$
\begin{aligned}
\left(\sum_{k=1}^{m-2}\right. & \left.+\sum_{k=m+2}^{\infty}\right)\left|p_{k} \log \right| \frac{F\left(z, a_{k}\right)}{F\left(z, a_{k}^{*}\right)}|| \\
& \leq \sum_{k=1}^{\infty} \frac{8 p_{k}\left(a_{k}^{*}-a_{k}\right)}{1-a_{k}^{*}} \leq \sum_{k=1}^{\infty} 8 \frac{2^{-k^{2}} 2^{k}}{\left(2^{-k}-2^{-k^{2}}\right) k^{2}}<C_{15}
\end{aligned}
$$

We note that (3.4) holds in the case when $r_{m-1} \leq|\zeta| \leq r_{m+2}$ and $|\varphi| \geq 1-r$ for $m \geq m_{0}$, and some $m_{0} \in \mathbb{N}$. Hence,

$$
\sum_{k=m-1}^{m+1} p_{k}\left|\log \frac{\left|F\left(z, a_{k}\right)\right|}{\left|F\left(z, a_{k}^{*}\right)\right|}\right| \leq \sum_{k=m-1}^{m+1} \frac{8 p_{k}\left(a_{k}^{*}-a_{k}\right)}{1-a_{k}^{*}}<C_{15} .
$$

Then, using (3.5) and (3.6), we deduce

$$
\begin{aligned}
& \left(\left.\frac{1}{2 \pi} \int_{1-r \leq|\varphi| \leq \pi}|\log | f\left(r e^{i \varphi}\right)\right|^{p} d \varphi\right)^{\frac{1}{p}} \\
& \quad \leq\left(\frac{1}{2 \pi} \int_{1-r \leq|\varphi| \leq \pi}\left(\sum_{k=1}^{\infty} p_{k}|\log | \frac{F\left(r e^{i \varphi}, a_{k}\right)}{F\left(r e^{i \varphi}, a_{k}^{*}\right)}||\right)^{p} d \varphi\right)^{\frac{1}{p}} \leq 2 C_{15} .
\end{aligned}
$$

In the case $|\varphi| \leq 1-r$ we write

$$
\log \left|F\left(z, a_{k}\right)\right|-\log \left|F\left(z, a_{k}^{*}\right)\right|=\log \left|\frac{z-a_{k}}{z-a_{k}^{*}}\right|+\log \left|\frac{1-z a_{k}^{*}}{1-z a_{k}}\right| .
$$

We have

$$
|\log | \frac{1-z a_{k}^{*}}{1-z a_{k}}||=|\log | 1-\frac{z\left(a_{k}^{*}-a_{k}\right)}{1-z a_{k}}|| \leq \frac{C_{16}\left(a_{k}^{*}-a_{k}\right)}{\left|1-z a_{k}\right|} \leq \frac{C_{17}\left(a_{k}^{*}-a_{k}\right)}{1-a_{k}}, \quad k \in \mathbb{N} .
$$

As above, we get $\sum_{k=1}^{\infty} p_{k}|\log | \frac{1-z a_{k}^{*}}{1-z a_{k}}||<C_{18}$.

To finish the proof we need a lemma.

Lemma 3.4. For any $a, b \in \mathbb{C}$, and $p>1$,

$$
\frac{1}{2 \pi} \int_{0}^{2 \pi}|\log | \frac{a-e^{i \varphi}}{b-e^{i \varphi}}||^{p} d \varphi \leq C(p)|a-b| .
$$

Proof of Lemma 3.4. Since we are going to apply the lemma in the case $|a-b|<1$, we omit the proof of the case $|a-b| \geq 1$ for simplicity.

We divide the unit circle into three parts:

$$
\begin{aligned}
& I_{1}=\left\{e^{i \theta}:\left|e^{i \theta}-b\right|>2|a-b|\right\} \cup\left\{e^{i \theta}:\left|e^{i \theta}-a\right|>2|a-b|\right\}, \\
& I_{2}=\left\{e^{i \theta}:\left|e^{i \theta}-b\right|<|a-b| / 2\right\} \cup\left\{e^{i \theta}:\left|e^{i \theta}-a\right|<|a-b| / 2\right\}, \\
& I_{3}=\partial \mathbb{D} \backslash\left(I_{1} \cup I_{2}\right) .
\end{aligned}
$$


Let $e^{i \theta} \in I_{1}$. Without loss of generality we may assume that $\left|e^{i \theta}-b\right|>2|a-b|$, $b=|b|$. Then

$$
\left|\frac{\left|e^{i \theta}-a\right|}{\left|e^{i \theta}-b\right|}-1\right| \leq \frac{|a-b|}{\left|e^{i \theta}-b\right|} \leq \frac{1}{2}
$$

Hence,

$$
\frac{1}{2 \pi} \int_{I_{1}}\left|\log \frac{\left|e^{i \theta}-a\right|}{\left|e^{i \theta}-b\right|}\right|^{p} \leq \frac{1}{\pi} \int_{\left|e^{i \theta}-b\right|>2|a-b|}\left(\frac{2|a-b|}{\left|e^{i \theta}-b\right|}\right)^{p} d \theta .
$$

For $J_{1}=\left\{e^{i \theta} \in I_{1}:|\theta|>|a-b|\right\}$, we have

$$
\frac{1}{\pi} \int_{J_{1}}\left(\frac{2|a-b|}{\left|e^{i \theta}-b\right|}\right)^{p} d \theta \leq \frac{\operatorname{mes}\left(J_{1}\right)}{\pi} \leq \frac{2|a-b|}{\pi}
$$

where $\operatorname{mes}\left(J_{1}\right)$ is the Lebesgue measure of the set $J_{1}$.

If $e^{i \theta}$ belongs to the complement of $J_{1}$ in $I_{1}$, we consider two subcases. If $|b| \geq \frac{1}{2}$, then standard estimates yield

$$
\begin{aligned}
& \frac{1}{2 \pi} \int_{I_{1} \backslash J_{1}}|\log | \frac{\left|e^{i \theta}-a\right|}{\left|e^{i \theta}-b\right|}||^{p} \\
& \quad \leq \frac{(2|a-b|)^{p}}{\pi}\left(\int_{|a-b|<|\theta| \leq \frac{\pi}{2}} \frac{d \theta}{(b|\sin \theta|)^{p}}+\int_{\frac{\pi}{2}<|\theta| \leq \pi} d \theta\right) \\
& \quad \leq \frac{(2|a-b|)^{p}}{\pi}\left(\pi^{p} \int_{|a-b|}^{\pi / 2} \frac{d \theta}{\theta^{p}}+\pi\right) \leq \frac{2^{p+1} \pi^{p-1}}{p-1}|a-b| .
\end{aligned}
$$

So, we have the required estimate for the integral over $I_{1}$.

We then consider the integral over $I_{2}$. We deduce

$$
\begin{aligned}
\int_{I_{2}} & |\log | \frac{e^{i \theta}-b}{e^{i \theta}-a}||^{p} d \theta \leq 2 \int_{\left|e^{i \theta}-b\right|<|a-b| / 2}|\log | \frac{e^{i \theta}-b}{e^{i \theta}-a}||^{p} d \theta \\
& \leq 2 \int_{\left|e^{i \theta}-a\right|<|a-b| / 2}\left(\log \frac{3|a-b|}{2\left|e^{i \theta}-b\right|}\right)^{p} d \theta \leq 2 \int_{\left|e^{i \theta}-b\right|<|a-b| / 2}\left(\log \frac{3|a-b|}{2|\sin \theta|}\right)^{p} \\
& \leq 2 \int_{|\theta|<|a-b|}\left(\log \frac{3 \pi|a-b|}{4|\theta|}\right)^{p} d t \leq 2|a-b| \int_{|\tau|<1}\left(\log \frac{3 \pi}{4 \tau}\right)^{p} d \tau=C_{19}(p)|a-b|
\end{aligned}
$$

Finally, if $e^{i \theta} \in \partial \mathbb{D} \backslash\left(I_{1} \cup I_{2}\right)$, then we have $|\log | \frac{e^{i \theta}-a}{e^{i \theta}-b}|| \leq 4$, and $\operatorname{mes}\left(\partial \mathbb{D} \backslash\left(I_{1} \cup\right.\right.$ $\left.\left.I_{2}\right)\right) \leq C_{20}|a-b|$. Therefore,

$$
\int_{\partial \mathbb{D} \backslash\left(I_{1} \cup I_{2}\right)}|\log | \frac{e^{i \theta}-b}{e^{i \theta}-a}||^{p} d \theta \leq C_{21}(p)|a-b| .
$$

The assertion of the lemma follows from (3.9)-3.12). 
We return to proving Theorem 3.3. Using Minkowski's inequality, the estimates (3.5), (3.6), and Lemma 3.4 we obtain, for $r_{m} \leq r \leq r_{m+1}$,

$$
\begin{aligned}
\left(\left.\frac{1}{2 \pi} \int_{|\varphi| \leq 1-r}|\log | f\left(r e^{i \varphi}\right)\right|^{p} d \varphi\right)^{\frac{1}{p}} & \\
\leq & \left(\frac{1}{2 \pi} \int_{|\varphi| \leq 1-r} \mid \sum_{k=m-1}^{m+1} p_{k}\left(\log \left|\frac{r e^{i \varphi}-a_{k}}{r e^{i \varphi}-a_{k}^{*}}\right|+\log \left|\frac{1-r e^{i \varphi} a_{k}^{*}}{1-r e^{i \varphi} a_{k}}\right|\right)\right. \\
& \left.\quad+\left(\sum_{k=1}^{m-2}+\sum_{k=m+2}^{\infty}\right) p_{k} \log \left|\frac{F\left(r e^{i \varphi}, a_{k}\right)}{F\left(r e^{i \varphi}, a_{k}^{*}\right)}\right|^{p} d \varphi\right)^{\frac{1}{p}} \\
\leq & \sum_{k=m-1}^{m+1} p_{k}\left(\left.\frac{1}{2 \pi} \int_{|\varphi| \leq 1-r}|\log | \frac{e^{i \varphi}-\frac{a_{k}}{r}}{e^{i \varphi}-\frac{a_{k}^{*}}{r}}\right|^{p} d \varphi\right)^{\frac{1}{p}}+\left(\frac{1}{2 \pi} \int_{|\varphi| \leq 1-r}\left(C_{18}+C_{15}\right)^{p} d \varphi\right)^{\frac{1}{p}} \\
\leq & C_{22} \sum_{k=m-1}^{m+1}\left(2^{k} k^{-2}\left(a_{k}^{*}-a_{k}\right)\right)^{\frac{1}{p}}+C_{23}(1-r)^{\frac{1}{p}} \\
\leq & C_{24}\left(\left(2^{-(m-1)^{2}+m} m^{-2}\right)^{\frac{1}{p}}+\left(1-r_{m}\right)^{\frac{1}{p}}\right)=o(1), \quad m \rightarrow+\infty .
\end{aligned}
$$

The last estimate together with (3.7) implies $m_{p}(r, f)=O(1)$ as $r \uparrow 1$ for any $p>1$.

3.2. Logarithmic derivative estimates. Results of this section allow us to obtain sharp lower estimates for the growth of solutions of linear differential equations in the unit disc. For this purpose one has to follow the scheme of the proof of Theorem 1.4 from [3]. However, it seems that neither the approach based on Herold's comparison theorem (see [7]) nor the Wiman-Valiron method give us sharp upper estimates for the $\rho_{\infty}$-order of solutions of linear differential equations in the most interesting case when $\rho_{M}<1$.

The following theorem is proved in [3].

Theorem F. Let $f$ be an analytic function in $\mathbb{D}$ such that $\rho_{M}[f]<\infty$. If $\rho_{M}[f]>$ 0 , let $\rho$ be a proximate order of $f$. Let $k$ and $j$ be integers satisfying $k>j \geq 0$, and let $\delta, \varepsilon \in(0,1)$. Then there exist an at most countable collection of discs $D_{\nu}=D\left(z_{\nu}, r_{\nu}\right)$, where $r_{\nu}<1-\left|z_{\nu}\right|$, and a constant $C>0$ such that

$$
\sum_{R<\left|z_{\nu}\right|<1} r_{\nu} \leq \delta(1-R), \quad R \uparrow 1,
$$

and

$$
\left|\frac{f^{(k)}(z)}{f^{(j)}(z)}\right| \leq \begin{cases}C\left(\frac{\log \frac{1}{1-|z|}}{\delta(1-|z|)^{\rho(|z|)+1}}\right)^{k-j}, & \text { if } \rho_{M}[f]>1 \\ \left(\frac{1}{\delta(1-|z|)}\right)^{2(k-j)+\varepsilon}, & \text { if } \rho_{M}[f] \leq 1\end{cases}
$$

for all $z \in \mathbb{D} \backslash \bigcup_{\nu} D_{\nu}$.

For a measurable set $E \subset[0,1)$, the upper linear density is defined as

$$
\mathcal{D}(E)=\limsup _{r \uparrow 1} \frac{\operatorname{mes}(E \cap[r, 1))}{1-r} .
$$


Corollary G. Under the assumptions of Theorem $\mathrm{F}$ there exists an exceptional set $E \subset[0,1)$ with $\mathcal{D}(E) \leq 2 \delta$ such that

$$
\left|\frac{f^{(k)}(z)}{f^{(j)}(z)}\right| \leq\left(\frac{1}{1-|z|}\right)^{\left(\max \left\{\rho_{M}[f], 1\right\}+1\right)(k-j)+\varepsilon}, \quad|z| \notin E .
$$

Since Theorem E and Corollary G are sharp in the sense that one cannot remove $\varepsilon$ in the exponent, it is not possible to obtain a better estimate than $O\left((1-|z|)^{-2(k-j)-\varepsilon}\right)$ in the same notation. However, the concept of $\rho_{\infty}$-order allows us to improve this estimate.

Theorem 3.5. Let $f$ be an analytic function in $\mathbb{D}$ such that $\rho=\rho_{\infty}[f]<\infty$. Let $k$ and $j$ be integers satisfying $k>j \geq 0$, and let $\delta, \varepsilon \in(0,1)$. Then there exist an at most countable collection of discs $D_{\nu}=D\left(z_{\nu}, r_{\nu}\right)$, where $r_{\nu}<1-\left|z_{\nu}\right|$, and a constant $C>0$ such that

$$
\sum_{R<\left|z_{\nu}\right|<1} r_{\nu} \leq \delta(1-R), \quad R \uparrow 1,
$$

and

$$
\left|\frac{f^{(k)}(z)}{f^{(j)}(z)}\right| \leq C\left(\frac{1}{\delta(1-|z|)^{\rho+1+\varepsilon}}\right)^{k-j},
$$

for all $z \in \mathbb{D} \backslash \bigcup_{\nu} D_{\nu}$.

Example 3.6. Let $f(z)=\exp \left\{-(1-z)^{-\alpha}\right\}$, where $\alpha>0, f(0)=1 / e$. Then $f$ is bounded if $\alpha \leq 1$, and hence $\rho_{M}[f]=0$. On the other hand, $\rho_{M}[f]=\alpha$ if $\alpha>1$. Besides, $\rho_{\infty}[f]=\alpha$ for all positive $\alpha$, and $\frac{f^{\prime}(z)}{f(z)}=-\frac{\alpha}{(1-z)^{\alpha+1}}$, which shows that estimate (3.17) is sharp in the sense that $\varepsilon$ cannot be removed.

The proof of Theorem 3.5 is similar to that of Theorem $\mathrm{F}$ [3. Define $\mathcal{A}_{1}=$ $\bar{D}\left(0, \frac{1}{2}\right)$ and the annuli $\mathcal{A}_{\nu}=\left\{\zeta: r_{\nu-1}<|\zeta| \leq r_{\nu}\right\}$ for $r_{\nu}=1-2^{\nu}, \nu \geq 2$. Then clearly $\mathbb{D}=\bigcup_{\nu} \mathcal{A}_{\nu}$. We now state and sketch the proof of an estimate (cf. Lemma 3 [3]), which is crucial in proving Theorem 3.5.

Lemma 3.7. Let $f$ be an analytic function in $\mathbb{D}$ such that $\rho=\rho_{\infty}[f]<\infty$. Let $\left\{a_{k}\right\}$ denote the sequence of zeros of $f$ listed according to multiplicities and ordered by increasing moduli, and let $0<\delta<1$. Then there exists an at most countable collection of discs $D_{\nu j}=D\left(z_{\nu j}, \rho_{\nu j}\right)$, where $\rho_{\nu j}<1-\left|z_{\nu j}\right|$, such that

$$
\sum_{\left|a_{k}\right| \leq r_{\nu+1}} \frac{1}{\left|z-a_{k}\right|} \leq \frac{C\left(\rho, R_{0}\right)}{\delta(1-r)^{\rho+1+\varepsilon}}, \quad z \in \mathcal{A}_{\nu} \backslash \bigcup_{j} D_{\nu j}
$$

where

$$
\sum_{R<\left|z_{\nu j}\right|<1} \rho_{\nu j} \leq \delta(1-R), \quad R \uparrow 1 .
$$

Proof of Lemma 3.7. Without loss of generality, we may assume that $\arg z=0$. Define $I_{s}=\left\{-2^{s}+1, \ldots,-1,0,1, \ldots, 2^{s}\right\}$ for $s \in \mathbb{N}$. For $\tau \in I_{s}$, define the polar rectangles $\mathcal{A}_{s \tau}=\left\{\zeta \in \mathcal{A}_{s}:(\tau-1) \pi 2^{-s} \leq \arg \zeta<\tau \pi 2^{-s}\right\}$.

Denote

$$
I_{s}^{*}= \begin{cases}I_{s}, & 1 \leq s \leq \nu-2 \\ I_{s} \backslash\{0,1\}, & \nu-1 \leq s \leq \nu+1 .\end{cases}
$$

With this notation, we have the following result [3, Lemma 4]. 
Lemma H. Let $\nu \geq 2,2 \leq s \leq \nu+1, \tau \in I_{s}^{*}, \zeta \in \mathcal{A}_{s \tau}, z \in \mathcal{A}_{\nu}$ and $\arg z=0$. Then

$$
|\zeta-z| \geq \begin{cases}\tau 2^{-s-1}, & \tau>0 \\ (|\tau|+1) 2^{-s-1}, & \tau \leq 0 .\end{cases}
$$

We return to the proof of Lemma 3.7. Let $n(U)$ denote the number of the points $a_{k}$ in a set $U \subset \mathbb{D}$. Recall that $\square\left(r e^{i \varphi}\right)=\left\{\zeta: r \leq|\zeta| \leq \frac{1+r}{2},|\arg \zeta-\varphi| \leq \frac{\pi}{4}(1-r)\right\}$. Then $\nu\left(r e^{i \varphi}\right) \leq C(1-r)^{-\rho-\varepsilon / 2}$.

If $z \in \mathcal{A}_{\nu}$ and $z \neq a_{k}$ for all $k$, write

$$
\begin{aligned}
\sum_{\left|a_{k}\right| \leq r_{\nu+1}} \frac{1}{\left|z-a_{k}\right|} & =\sum_{s=1}^{\nu+1} \sum_{\tau \in I_{s}} \sum_{a_{k} \in \mathcal{A}_{s \tau}} \frac{1}{\left|z-a_{k}\right|} \\
& \leq \sum_{s=1}^{\nu+1} \sum_{\tau \in I_{s}^{*}} \sum_{a_{k} \in \mathcal{A}_{s \tau}} \frac{1}{\left|z-a_{k}\right|}+\sum_{s=\nu-1}^{\nu+1} \sum_{\tau=0}^{1} \sum_{a_{k} \in \mathcal{A}_{s \tau}} \frac{1}{\left|z-a_{k}\right|} \\
& =\sum_{1}+\sum_{2} .
\end{aligned}
$$

To deal with the sum $\sum_{1}$ in (3.20), we first observe that $r_{s}=\frac{1+r_{s-1}}{2}$ and $\tau \pi 2^{-s}-(\tau-1) \pi 2^{-s}=\pi 2^{-s}=2 \pi 2^{-2}\left(1-r_{s-1}\right)$. Lemma $\mathrm{H}$ now yields

$$
\begin{aligned}
\sum_{1} & \leq \sum_{s=1}^{\nu+1} \sum_{\tau \in I_{s}^{*}} \frac{n\left(\mathcal{A}_{s \tau}\right)}{\inf _{\zeta \in \mathcal{A}_{s \tau}}|z-\zeta|} \\
& \leq \sum_{s=1}^{\nu+1}\left(\sum_{\tau \in I_{s}^{*}, \tau>0} \frac{n_{1}\left(r_{s-1}\right)}{\tau} 2^{s+1}+\sum_{\tau \in I_{s}^{*}, \tau \leq 0} \frac{n_{1}\left(r_{s-1}\right)}{|\tau|+1} 2^{s+1}\right) \\
& \leq 8 \sum_{s=1}^{\nu+1} \frac{n_{1}\left(r_{s-1}\right)}{1-r_{s-1}}\left(\sum_{\tau=1}^{2^{s}} \frac{1}{\tau}\right) \\
& \leq 8(1+(\nu+1) \log 2) \sum_{s=1}^{\nu+1} \frac{n_{1}\left(r_{s-1}\right)}{1-r_{s-1}} \leq 24 \nu \sum_{s=1}^{\nu+1} 2^{(s-1)(\rho+1+\varepsilon)} \\
& <48 \nu 2^{\nu(\rho+1+\varepsilon / 2)}<\frac{C}{\left(1-r_{\nu}\right)^{\rho+1+\varepsilon}} .
\end{aligned}
$$

To deal with the sum $\sum_{2}$ in (3.20), define

$$
U=\bigcup_{s=\nu-1}^{\nu+1} \bigcup_{\tau=0}^{1} \mathcal{A}_{s \tau}, \quad N_{\nu}=n(U), \quad \text { and } \quad \delta_{\nu}=\delta \cdot 2^{-\nu-6}
$$

Then, by the Cartan lemma 8, pp. 19-21], there exists a finite collection of discs $D\left(w_{\nu j}, h_{\nu j}\right)$ with $\sum_{j} h_{\nu j}=2 \delta_{\nu}$ and a permutation of $\left\{a_{k}\right\} \subset U$, say $b_{1}, \ldots, b_{N_{\nu}}$, such that $\left|z-b_{m}\right|>m \delta_{\nu} / N_{\nu}$ for all $m=1, \ldots, N_{\nu}$ and $z \notin \bigcup_{j} D\left(w_{\nu j}, h_{\nu j}\right)$. Hence, by noting that

$$
n\left(\mathcal{A}_{s \tau}\right) \leq n_{1}\left(r_{s-1}\right), \quad s=\nu-1, \ldots, \nu+1, \tau=0,1
$$


it follows that

$$
\begin{aligned}
\sum_{2} & =\sum_{a_{k} \in U} \frac{1}{\left|z-a_{k}\right|}=\sum_{m=1}^{N_{\nu}} \frac{1}{\left|z-b_{m}\right|} \\
& \leq \frac{N_{\nu}}{\delta_{\nu}} \sum_{m=1}^{N_{\nu}} \frac{1}{m} \leq \frac{2^{5} N_{\nu}}{\delta\left(1-r_{\nu+1}\right)}\left(1+\log N_{\nu}\right) \\
& \leq \frac{C}{\delta} \sum_{s=\nu-2}^{\nu+1} \frac{n_{1}\left(r_{s}\right)}{1-r_{s}} \log n_{1}\left(r_{s}\right) \leq \frac{C}{\delta\left(1-r_{\nu}\right)^{\rho+1+\varepsilon}}
\end{aligned}
$$

for all $z \in \mathcal{A}_{\nu}$ such that $z \notin \bigcup_{j} D\left(w_{\nu j}, h_{\nu j}\right)$.

By combining (3.20)-3.22) we conclude that

$$
\sum_{\left|a_{k}\right| \leq r_{\nu+1}} \frac{1}{\left|z-a_{k}\right|} \leq \frac{C\left(\rho, R_{0}\right)}{\delta(1-r)^{\rho+1+\varepsilon}}
$$

for all $z \in \mathcal{A}_{\nu}$ such that $z \notin \bigcup_{j} D\left(w_{\nu j}, h_{\nu j}\right)$.

An estimate of the exceptional set repeats that given in [3].

\section{ACKNOWLEDGEMENTS}

The author would like to thank Prof. B. N. Khabibullin, who suggested that he consider a ratio of Blaschke products in order to answer Question 3.2. The author also thanks Dr. J. Rättyä and the anonymous referee for valuable remarks.

\section{REFERENCES}

1. I. E. Chyzhykov, On a complete description of the class of functions without zeros analytic in a disk and having given orders, Ukrainian Math. J. 59 (2007), no. 7, 1088-1109. MR2397653 (2008j:30068)

2. I. E. Chyzhykov, Growth of analytic functions in the unit disc and complete measure in the sense of Grishin, Mat. Stud. 29 (2008), no. 1, 35-44. MR2424597(2009e:30062)

3. I. Chyzhykov, J. Heittokangas and J. Rättyä, Sharp logarithmic derivative estimates with applications to differential equations in the unit disc, J. Australian Math. Soc. 88 (2010), no. 2, 145-167. MR2629927 (2011f:30064)

4. M. M. Džrbašjan, Integral transforms and representations of functions in the complex domain, Moscow, Nauka, 1966 (in Russian). MR0209472(35:370)

5. A. Grishin, On regularity of the growth of subharmonic functions. III, Teorija Funktsii Funktsionalnyj Analiz i ih Prilozh. (1968), no. 7, 59-84 (in Russian).

6. O. P. Juneja and G. P. Kapoor, Analytic Functions - Growth Aspects, Research Notes in Mathematics 104, Pitman Adv. Publ. Prog., Boston-London-Melbourne, 1985.

7. J. Heittokangas, R. Korhonen and J. Rättyä, Growth estimates for solutions of linear complex differential equations, Ann. Acad. Sci. Fenn. Math. 29 (2004), 233-246. MR2041951 (2004m:34199)

8. B. Ja. Levin, Distribution of zeros of entire functions (revised edition, Transl. Math. Monographs, Volume 5, translated by R. P. Boas et al.), Amer. Math. Soc., Providence, 1980. MR589888 (81k:30011)

9. C.N. Linden, The representation of regular functions, J. London Math. Soc. 39 (1964), 19-30. MR0160910(28:4119)

10. C.N. Linden, On a conjecture of Valiron concerning sets of indirect Borel points, J. London Math. Soc. 41 (1966), 304-312. MR0192056 (33:283)

11. C. N. Linden, Integral logarithmic means for regular functions, Pacific J. of Math. 138 (1989), no. 1, 119-127. MR.992177 (90g:30035)

12. C. N. Linden, The characterization of orders for regular functions, Math. Proc. Cambridge Phil. Soc. 111 (1992), no. 2, 299-307. MR.1142749 (93a:30043) 
13. A. G. Naftalevich, On interpolation of functions meromorphic in the unit circle, Dokl. Akad. Nauk SSSR 88 (1953), no. 2, 205-208 (in Russian). MR0054026 (14:858h)

14. M. Tsuji, Potential theory in modern function theory, Chelsea Publishing Co. Reprinting of the 1959 edition. New York, 1975. MR0414898 (54:2990)

15. M. Tsuji, Canonical product for a meromorphic function in a unit circle, J. Math. Soc. Japan 8 (1956), no. 1, 7-21. MR0103273 (21:2051)

Faculty of Mechanics and Mathematics, Ivan Franko National University of Lviv, UNIVERSYTETS'KA 1, 79000, LVIV, UKRAINE

E-mail address: chyzhykov@yahoo.com 